\title{
The Spherical Visibility Map
}

\author{
Todd Keeler, John Fedorkiw, and Sherif Ghali
}

\begin{abstract}
We introduce a novel representation for visibility in three dimensions and describe an efficient algorithm to construct it. The data structure is a spherical map that consists of a doubly-connected edge list embedded on the surface of a sphere. Each face of the spherical map is labeled with the polygon visible in the corresponding cone. We demonstrate that the algorithm is efficient and robust by showing statistics of its time and space requirements for handling several classes of input.
\end{abstract}

\section{Introduction}

Vector visibility is a fundamental problem in Computer-Aided Design in particular and in computing in general. The problem is to find, given a set of polygons and an observer in 3D, the set of polygons visible from the observer, where a point on a polygon is visible from the observer if and only if the line segment joining them does not intersect any other polygon. If the output is meant for display on a raster device, then the problem becomes significantly simpler as only a set of samples need be computed.

An inspection of the drawings produced in books and journals, which can and wish to maintain the highest possible print quality, reveals that many of the rendered images are raster images. Raster visibility is in fact casually used for computing images that will be embedded in Postscript (or Adobe Acrobat) files, while the Postscript language was specifically designed for faithfully producing vector images. One would suspect that this is a consequence that despite years of research on both the theoretical and the practical sides of computing a 2D vector image of a 3D scene, no satisfying data structure and algorithm for solving the problem are known. Work on vector visibility is easily attacked. If the resolution of the rendered image is insufficient, simply recompute a new image at a higher resolution, is how the argument goes. Such a claim misses the point. We wish to represent visibility in a structure that captures the answer for any desired resolution as well as the (now largely obsolete) vector printing devices such as pen plotters.

Vector images have many advantages. Knowing the answer to visibility at a large number of sample points (or pixels) tells us little about the structure of the image. The size of a vector image can be arbitrarily concise compared to that of a raster image of no preset resolution. Vector images can be easily manipulated and are often the only format that can be reasonably embedded in a work flow. The structure of the image is revealed by a planar graph representing visibility, which affords further potential for processing. Vector images can also be easily converted into raster images, whereas the opposite conversion is difficult and illdefined. Yet several major 3d modeling systems (geared to the entertainment industry) completely omit an option for producing vector output and other major $3 \mathrm{~d}$ modeling systems (geared to the CAD industry) have an option for producing vector output that can be made to produce an incorrect result.

A projective transformation has traditionally preceded the computation of vector visibility, but even though such a transformation is needed before the computation of raster images, no such transformation is needed for the computation of vector images; such images are most naturally embedded on a sphere. It is significantly easier to perform sweeping on a plane than on a sphere and since sweeping has frequently been

Computer-Aided Design, Volume 39, Issue 1, January 2007, Pages 17-26 
the method of choice for vector visibility, all prior work has assumed the viewer is located at infinity and the projection is orthographic. This work diverges - it generates instead a vector view consisting of a set of spherical polygons bounded by spherical segments and spherical points. Each spherical point is described geometrically by a vector in three-space. There are several advantages to using spherical projection. By embedding a vector image on the surface of a sphere centered at the observer and constructing a spherical map, any perspective image seen by the observer can subsequently be constructed. Vector images on a view plane, arbitrarily sampled images, perpendicular or oblique projections are all subsumed and can be determined from a spherical map. A spherical map is also an ideal structure to capture visibility from the maximal set of points in space that share the same qualitative image and may thus find many practical applications in computer vision. It is unnecessary to specify the limited field of view that results from using a perspective transformation. The view in all directions can be simultaneously computed and represented.

The main drawback has been that algorithms for hidden surface removal/vector visibility are either efficient, but so intricate that no one has so far attempted to implement them ${ }^{1}[39,14]$ or that they have been practical, but lack efficiency, versatility, or robustness to be used as a general-purpose practical solution $[1,49,16,37,28,33]$. Our algorithm has been implemented, is fully robust, and exhibits sub-quadratic complexity for useful classes of input.

A word about the terminology is in order. The classical characterization paper [53] so influenced the subsequent nomenclature that object-space algorithms has been the standard term to describe the class of algorithms that operate without rasterization. Since many algorithms that generate raster output (such as ray tracing) operate in object space, this term has been criticized [22, footnote, page 650] and the alternative object precision has been suggested. We use here the original and unambiguous vector qualifier, which is also widely used in the related discipline of Scalable Vector Graphics.

The machinery we use for the computation of the spherical visibility map relies on several ideas in the literature. Warnock used divide-and-conquer to compute visibility [58]; Weiler and Atherton used front-toback sorting and described a general clipping algorithm [59]; Chin and Feiner used BSP trees for shadow computation [11]; and Thibault and Naylor [55, 56, 41] showed how to compute Boolean operations on regular sets. The regular sets in our case are spherical polygons embedded on a sphere. We maintain a recursive partitioning of the sphere and an accompanying planar graph embedded on the sphere. Interconnectivity is maintained for all topological structures through a Doubly-Connected Edge List (DCEL) [7, 38, 30].

In Section 2 we survey practical and theoretical previous work. Section 3 contains the description of our algorithm, its implementation, and runtime experiments. Section 4 concludes the paper by suggesting future work.

\section{Previous Work}

A large number of hidden surface removal/vector visibility algorithms have appeared in both theoretical and practical venues. But theoretical and practical results serve different purposes and can in general not be compared. We discuss in what follows the main ideas that have been used for vector visibility. The interested reader will find elsewhere an extensive survey [18], which also compares the algorithms for special classes of input (e.g. terrains).

The first published algorithm computes hidden line removal by solving a linear program for each edge in the scene [45]. Appel subsequently observes that if the number of polygons that occlude one vertex of a polyhedron is known, then that number, the vertex's quantitative invisibility, can be updated along each edge

\footnotetext{
${ }^{1}$ We have ourselves attempted to implement one of the two algorithms [39], and failed. We speculate that no developer, of any skill, could produce an implementation of the second algorithm [14].
} 
of the polyhedron [1]. Optimizations of this idea for various inputs are known [26, 34, 44], but even though at the outset quantitative invisibility appears simple as it suffices to use two nested loops, in practice many subtleties make it difficult to produce a correct implementation [8]. Appel's algorithm takes $O\left(n^{2} \log n\right)$ time for a scene consisting of $n$ edges.

Several mixed vector-raster algorithms are known. The classical back-to-front painter-style rendering once a depth order is known produces a raster image [43]. A superior approach is to produce raster images by performing front-to-back rendering [29] since shading then only needs to be computed at visible pixels. A third hybrid algorithm, Warnock's [58], is the first to use recursion to compute visibility using a quadtreelike partitioning of the image plane. Franklin's algorithm uses the same notion of reducing polygon-polygon comparisons through subdivision, but his algorithm outputs a set of polygons [24].

A number of utilities have been described for converting from OpenGL to Postscript. Such utilities operate by either sorting using the barycenter of a set of triangles and painting from back to front or by computing a depth order using a BSP tree before painting. The barycenter sorting approach fails easily if, say, a small triangle close to the viewer is hidden by a larger triangle; the barycenter of the small triangle will indeed be closer, but it should be drawn first, not second. The BSP approach may be quite satisfactory for some applications, but the Postscript output still contains all hidden primitives and the expense of computing occlusion will be borne by the Postscript engine. More importantly, the computation will be performed in the raster domain and so the structure of the image is never recovered. We have implemented variations on the ideas described above and can report that the simple argument of triangulating a scene to minimize the size of the input triangles does not work; some inputs will inherently render incorrectly.

We illustrate a third tempting approach, but one that will also fail for some inputs. The idea is also a hybrid raster-vector approach, but rather than performing the vector computation first, the raster computation is performed first, which would conveniently make it possible to use visibility culling approaches. Once the raster image is constructed, one scans each pair of adjacent pixels and constructs a graph in which an edge is recorded between two polygons if one occludes the other. The argument is that the transitive closure of the graph would constitute a valid back to front sorting of only the visible polygons. Even if we forego the inadequacy of this approach for not providing the structure of the vector image, it is still unsuitable since it can fail on inputs such as the one shown in Figure 1. An edge is added in the graph shown in the right of the figure between two polygons if one occludes the other. The shaded region describes an area that could not be resolved by this technique; the graph has no information to depth order the two regions $f$ and $c$. The problem is not any easier if the scene consists solely of convex polygon since a heuristic must then be found to determine depth relationships between pairs of adjacent and non-overlapping polygons.

Weiler and Atherton designed the first algorithm that performs vectorial front-to-back clipping [59]. Their algorithm maintains a mask describing the union of the polygons processed so far and takes $O\left(n^{3}\right)$ time in the worst case.

Dévai and McKenna designed two algorithms $[15,37]$ that are asymptotically superior to Appel's, taking time $\Theta\left(n^{2}\right)$, but both algorithms start by extending the projections of scene edges into lines and then operate on the resulting arrangement and thus neither is likely to be more efficient than Appel's algorithm in practice.

Others have also described algorithms that compute the arrangement of the projection of scene edges. Schmitt and Goodrich compute the necessary intersections. If the number of such intersections is $k$, Schmitt's algorithm takes time $O((n+k) \log n)$ and Goodrich's takes time $O(n \log n+k+t)$, where $t$ is the number of polygon-polygon overlaps on the view plane [46, 47, 28]. Their algorithms do achieve intersection sensitivity - unnecessary edge intersections are never computed, but using them in practice is not easy because designing and implementing a segment sweep algorithm that handles all degeneracies is itself a significant challenge [6].

It is desirable that a visibility algorithm not spend time on the hidden features of the scene. This ob- 

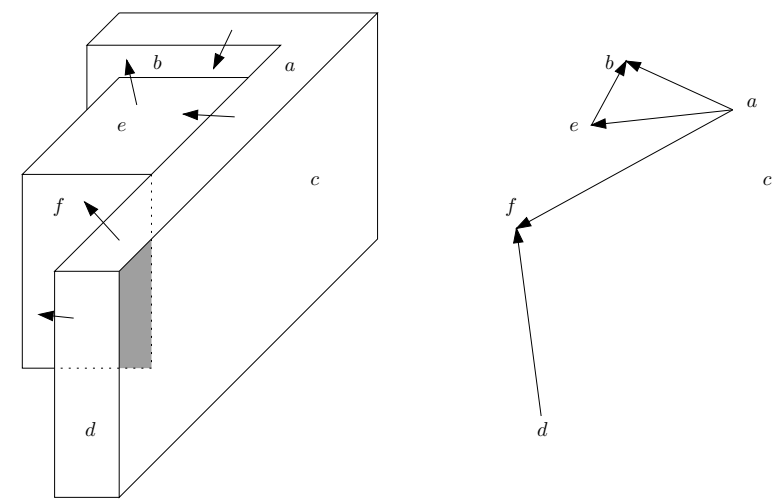

Figure 1: Topologically sorting a neighborhood overlap graph fails to construct visible surfaces for some classes of input.

jective, output sensitivity, has been almost achieved by Mulmuley [39]. The time spent by his algorithm to eliminate the intersection of a pair of hidden edges decreases as the invisibility, or the number of polygons occluding the intersection, increases. Another output-sensitive algorithm takes time $O\left(n^{1+\epsilon} \sqrt{d}\right)$ to produce an output of size $d[14,13]$. No report of an implementation for either algorithm has so far appeared.

Several methods use beam tracing as a vectorial generalization of ray casting [31, 12, 23, 57]. The most potent beam tracing methods rely on variations of divide-and-conquer, which first appeared in the literature in the guise of binary partitioning [48], but, significantly, without the explicit construction of an associated binary tree [25]. In addition to their traditional use for depth order computation, BSP trees can also be used for Boolean operations on point sets in an arbitrary dimension [56], for shadow computation [11], and for representing polytopes [40]. Binary trees have been similarly used to compute visibility [32, 51]. BSP trees are now a classical method for estimating the potentially visible set, a conservative estimation of the polygons visible from a "cell" in the scene [54] that is widely used in game applications.

One of the major challenges of algorithms with vector output is their robustness. The description in Sections 3 and 3.3 show how to handle all cases of degenerate input. Extensive experiments with both random and non-random inputs suggest that the algorithm is robust.

\section{Binary Partitioning Yields Spherical Visibility}

Let $S$ be a scene in 3D consisting of a set of piecewise linear polyhedra and let $O$ be the location of an observer outside $S$. Our objective is to compute a spherical partition $\mathcal{T}$ of the polygons in $S$ visible from $O$ as they would project on the sphere of directions $S^{2}$ centered at $O$. A direction is a unit vector, although in practice a normalization step is not necessary.

\subsection{Spherical Geometry}

We briefly review a few elements from spherical geometry and give the outline for implementing spherical geometry classes. A spherical point $P$ on $S^{2}$ is described by a direction $\vec{D}$. The point $Q$ antipodal to $P$ is defined by the direction $-\vec{D}$. An oriented spherical circle $C$ is a great circle on $S^{2}$ and is defined by the normal $\vec{N}$ to an oriented plane in 3D passing through the center of the sphere. It is then possible to speak of the open positive and the open negative hemispheres defined by $C$. They lie, respectively, on the positive and on the negative side of the plane passing by $O$ with normal $\vec{N}$. A point is on the positive side 
of an oriented circle $C$ if it lies to the left of an observer walking along $C$ on the outside of the sphere. See Figure 2.

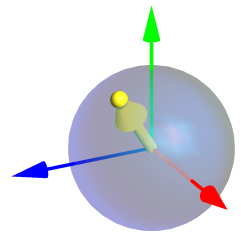

(a)

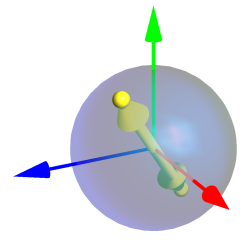

(b)

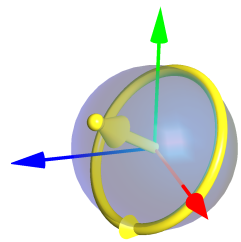

(c)

Figure 2: (a) A spherical point. (b) The antipodal point $Q=-P$ of a point $P$. (c) An oriented spherical circle

The only object needed to implement a class for a spherical point is a vector in three dimensions. It is sufficient to remember while implementing the member functions that such a vector is meant to be a unit vector, but it is not necessary to actually perform the normalization. Avoiding the normalization is important for more than efficiency; rational numbers can then be used as the underlying number type. One exception to normalization avoidance is the visualization step, but at that stage the coordinates need in any case be converted to floating point numbers to interface with the graphics library.

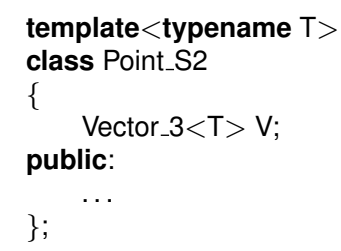

A spherical circle is in turn defined by a spherical point. A predicate such as the sidedness of a spherical point with respect to a spherical circle can then be computed by determining the sign of the dot product of the two vectors.

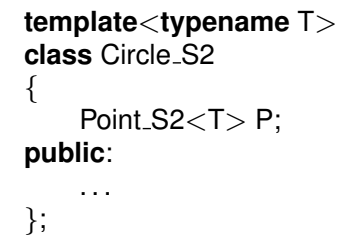

A spherical segment is a continuous subset of a spherical (or great) circle and so two distinct nonantipodal points would suffice to determine a spherical segment. Yet it is convenient to represent a spherical segment by its end spherical points as well as by the spherical circle on which the segment lies. Two (noncoincident and non-antipodal) spherical points define as many as four different spherical segments. The four spherical segments lie on the spherical (great) circle defined by the two points and arise because there are two ways to order the points and two ways to orient the spherical circle. The option shown in Figure 3 illustrates that it is convenient to use a spherical circle in addition to the (ordered) pair of spherical points to define a spherical segment. A spherical segment is oriented and the set of points on the segment is defined such that a counter-clockwise turn on the spherical circle leads from the source to the target of the segment. 
Since the spherical circle is specified independently and is not deduced from the two spherical points, it is not necessary to exclude the case of the two spherical points being antipodal, though the two points still cannot be coincident since it would otherwise be necessary to add a fourth parameter identifying whether the full circle or a single point is intended. But if either a point or a circle is indeed intended, the appropriate class should be used instead.

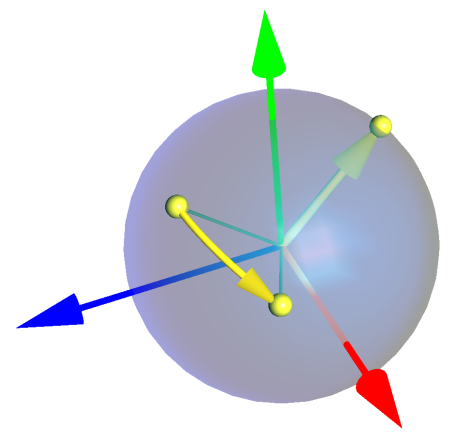

Figure 3: A spherical segment is defined by two spherical points in addition to an independently specified spherical circle.

A sketch of an implementation for a spherical segment class is shown below. If the spherical circle is chosen not to be stored, then an predicate such as sidedness can be implemented by reduction to a $3 \times 3$ determinant. If the code below is used, sidedness is implemented by evaluating the dot product of the vector defining the spherical circle and the spherical point in question.

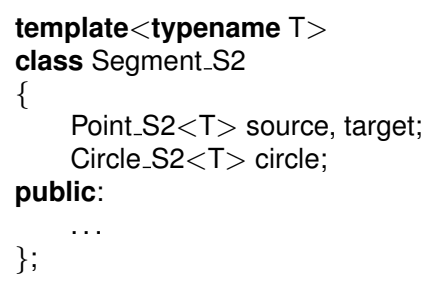

A spherical polygon on $S^{2}$ is described by a sequence of spherical circles defining a closed loop; while traversing the circles in order, the polygon lies on the left as seen from $O$. See Figure 4. Since two spherical points result from the intersection of two consecutive spherical circles, it is not possible (or desirable) to use spherical objects for the intersection calculation. Each spherical circle is stored along with the scene segment that defines it and the intersection is performed by determining the common line passing by the center of projection and the two scene segments.

We illustrate one subtlety of implementing the algorithm by describing first the flaw of using the obvious method of representing a spherical polygon. For simplicity we use a triangle for illustration. Consider that a spherical triangle is represented using three spherical points. Consider further that the spherical triangle is split by some spherical circle. If the two resulting fragments are defined using the original spherical points in addition to the two spherical points obtained from splitting, it will be impossible to process very large scenes and it will be impossible to guarantee the robustness of the algorithm. The difficulty is that these new spherical points would subsequently be used to define new spherical circles that are in turn used for 


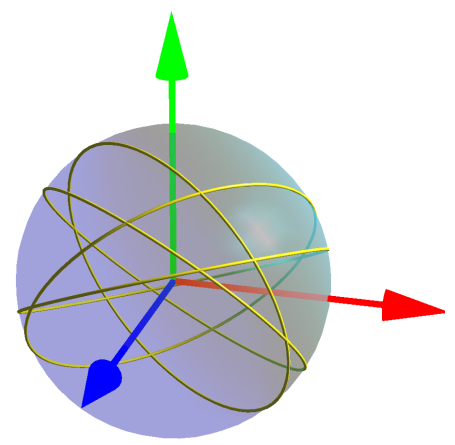

Figure 4: A spherical polygon is defined by a sequence of spherical circles.

splitting. Such a derivation of this and any geometric algorithm suffers from the use of predicates of high algebraic degree $[4,5]$. In essence we wish that the numbers (floating or otherwise) used for evaluating predicate be obtained using as few manipulations as possible from the input data numbers. In our case the depth of the arithmetic expression could be as deep as the depth of the tree used for computing visibility. If one uses floating point numbers, this results in a compounding of errors and the reliability of the floating point numbers quickly diminishes. If one uses rational numbers with filtering, the number of bits used to represent the numbers grows proportionally to the depth of the arithmetic expression computing them, which means that the rational numbers become large and that computing predicates based on them becomes slow. Using floating point filtering does not help, since operations such as determining the incidence between a spherical point and a spherical circle are needed often enough for the filter to fail and for the computation of the rational expression to be needed.

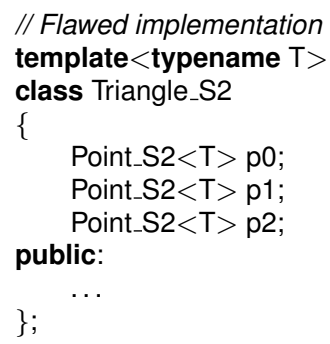

We conclude that a spherical triangle must be defined using the three bounding spherical circles. By extension, a spherical polygon must be defined using a sequence of spherical circles. As discussed, in practice the lines in space carrying the scene edges resulting in the spherical circles need to be stored along with the spherical circles since it would be otherwise impossible to disambiguate between the two spherical points obtained from the intersection of two spherical circles.

\subsection{The Spherical Partition}

The spherical partition $\mathcal{T}$ covers $S^{2}$ and captures the visibility of the scene as seen from the observer $O$. $\mathcal{T}$ is stored using a DCEL, which consists of a set of nodes, arcs, and faces. These three sets of elements cross-reference each other such that it is possible, for example, to list the faces adjacent to a given node or 


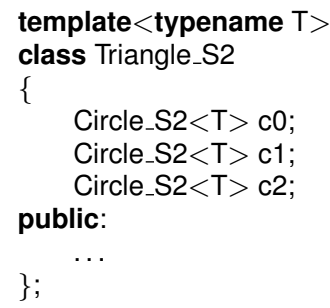

those adjacent to a given face. All elements of the DCEL are embedded on $S^{2}$ : A node stores a spherical point in addition to either the scene vertex projecting onto it or the pair of edges that, along with $O$, have a common line stabber in 3D in that direction. Each face in $\mathcal{T}$ stores either a pointer to the polygon in $S$ that is (partially) visible along the face's directions or a null pointer if no polygon is visible.

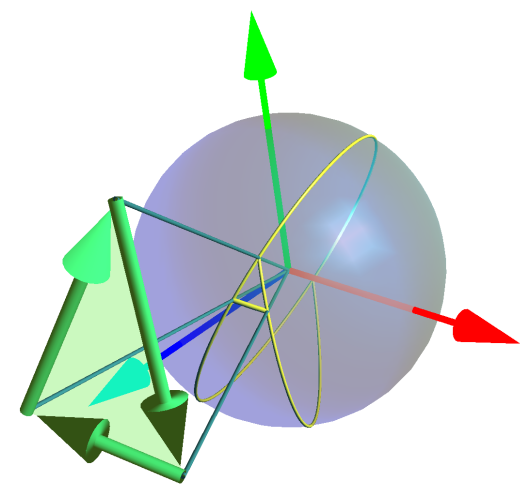

Figure 5: Each of the three lines carrying the segments of a triangle defines, along with the center of projection, a plane. Each plane defines a spherical circle.

A secondary BSP tree on the polygons in $S$ is built and queried with the location of the observer, resulting in a depth-ordered list of the polygons in $S$. Each polygon is extracted in front-to-back order from the observer's position and is handled incrementally. Polygons that are either backfacing with respect to $O$ or whose carrying plane passes by $O$ are discarded. Each edge of a front-facing polygon projects to a segment on $S^{2}$ and the sequence of edges for one polygon defines a polygon on $S^{2}$.

A binary partitioning tree $\mathcal{V}$ is used both to be able to determine efficiently the faces of $\mathcal{T}$ where a newly inserted polygon lies, as well as to partition the new polygon into fragments such that each fragment projects on exactly one face of $\mathcal{T}$ corresponding to one leaf node of $\mathcal{V}$. Each interior node of $\mathcal{V}$ stores a partitioning plane and each leaf node stores a pointer to the corresponding face in $\mathcal{T}$. $\mathcal{V}$ is initialized to a single node corresponding to the entire sphere $S^{2}$.

Figure 6 shows $\mathcal{V}$ (left) and $\mathcal{T}$ (right) as the first polygon (a triangle in the figure) is handled. Figure 5 shows the three edges of the triangle that, along with $O$, determine three planes used to partition $S^{2}$ recursively.

Each polygon is thus inserted into $\mathcal{V}$ and the partitioning plane at the root of $\mathcal{V}$ is used to split the polygon. The resulting one or two fragments are recursively inserted in the two children of the node. When a polygon eventually reaches a leaf node, the corresponding face of $\mathcal{T}$ is tested. If it is already marked with a polygon fragment, the polygon is hidden and can be discarded. Otherwise (the remaining fragment of) the 


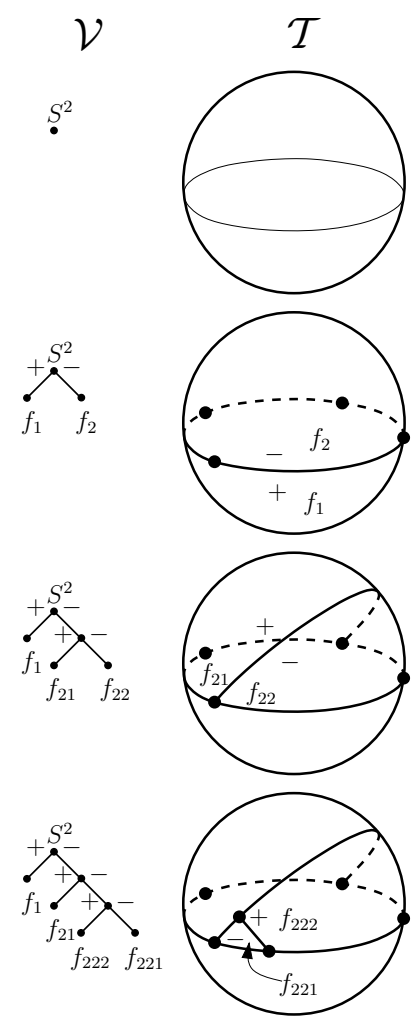

Figure 6: The spherical partition $\mathcal{T}$ embedded on $S^{2}$ and the BSP tree $\mathcal{V}$.

polygon is known to be visible from $O$.

The polygon does not necessarily occupy the entire cell in $\mathcal{T}$ and here the addition of a new subtree becomes a delicate operation that must be implemented carefully to ensure robustness. The desired constraint is to guarantee that any path from the root to a leaf in $\mathcal{V}$ not contain duplicate partitioning planes. This constraint in turn ensures that there are no degenerate nodes in $\mathcal{V}$, or ones that occupy zero surface on $S^{2}$.

To satisfy this constraint we store a Boolean flag with each edge of each polygon. Initially all flags are set to true, indicating that each edge would, along with $O$, construct a node in $\mathcal{V}$. When a polygon is split by a partitioning plane, each edge maintains the flag it had prior to partitioning, but the new edges are assigned a flag of false, indicating that a partitioning plane is already present in the tree in the path taken during their insertion. The polygon clipping routine must also detect the case a polygon is not split by a partitioning plane, but one of its edges lies in the plane. The flag of that edge must then be reset to false. This scenario arises often since the input will most likely not consist of individual polygons, but of a mesh of polygons. Once one polygon is inserted in $\mathcal{V}$, all its edges will appear in $\mathcal{T}$ and there is no longer a need to insert these edges for any of the adjacent polygons in the input mesh. In the base case a fragment of a polygon reaches a leaf node of $\mathcal{V}$. Only those edges whose flags have not be reset are used to define a subtree at that node.

When a face $f$ of $\mathcal{T}$ is split by a partitioning plane, we are certain that the spherical circle on $S^{2}$ splits the face $f$ in its interior. We search for the two spherical segments of $f$ that intersect the circle. If the circle intersects a segment in its interior, that segment is split by inserting a node at the location of the intersection and executing the splitEdge Euler operator [36]. If the circle passes by a node of $f$, no additional node is inserted. Once the two (possibly new) nodes are determined, the face $f$ is split by adding an edge between these two nodes using the Euler operator make-edge-face (mef). See Figure 7. 


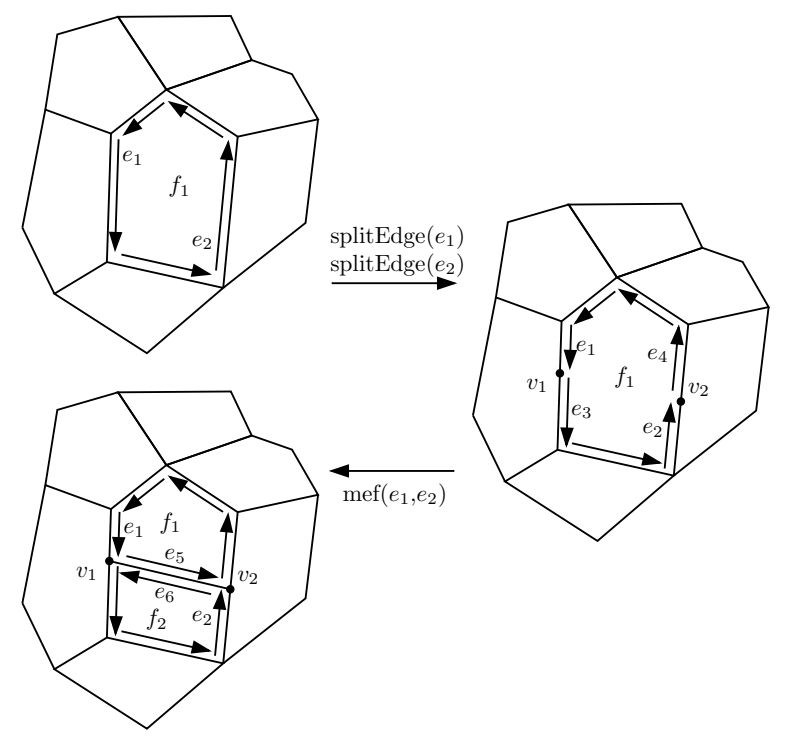

Figure 7: The two Euler operators splitEdge and mef suffice to construct the spherical map incrementally.

\subsection{Implementation and Results}

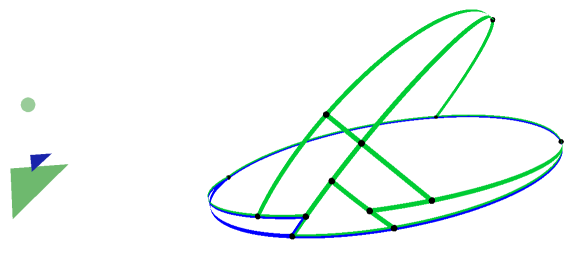

(a)
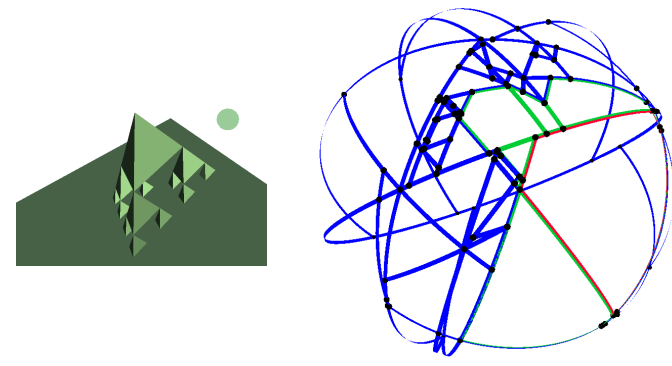

(b)

Figure 8: (a) The spherical visibility map for the simple scene shown on the left. Even though only the polygons visible from the observer at the center of the sphere are shown in the spherical map, it appears that some hidden lines have not been removed. This results because we project both the "near" and the "far" sides of the sphere on the image (paper) plane. Another way of stating this is that the image on the left represents the view of a view. (b) One polygon is highlighted in red in the spherical visibility map shown on the right and the polygons adjacent to that polygon are highlighted in green. In practice one would be less interested in visualizing the spherical visibility map itself, but would be more interested in coupling the data structure with another projection algorithm with a suitable user interface.

The algorithm has been implemented and extensive tests suggest that it is robust. The geometric kernel of CGAL $[20,10]$ provides the classes for points, vectors, and other elementary geometric objects and its topological maps [21] represent the spherical map $\mathcal{T}$. The GLOW [3] library provides an implementation of the Model-View-Controller design patterns in its user interface classes and also encapsulates a trackball module to rotate the spherical map and view the projection of the scene. Using these layers of geometric software simplified the effort needed for an implementation. Figure 8 shows two examples of input scenes 
and the resulting spherical maps. The user interface locates the face on the sphere where the user clicks and the system subsequently highlights the adjacent faces from the adjacency information in the DCEL.

The DCEL we use [21] is meant for a planar map embedded on a plane and so it is not possible to insert the first partitioning plane (an edge in the map) without special handling. When this first partitioning plane is determined, we find its two basis vectors (any pair of orthogonal vectors lying in it are suitable) and use the two vectors to define two spherical points. The two points and their antipodal points are used to define the root partition of $S^{2}$ as shown in Figure 6.

We implemented a robust polygon clipping algorithm that handles all special cases and that simultaneously generates the fragments on both sides of the partitioning plane. The algorithm is an extension to Sutherland and Hodgman reentrant polygon clipping algorithm [52]. We chose to keep the implementation simple by handling only convex polygons, which still makes it possible to handle concave solids.

We give time and space benchmarks for the result of running the algorithm on various inputs. The following four scenes, each a representative sample of a class of input, were used in various sizes in our experiments. The inputs are perhaps contrived, but are important nevertheless. The representative scenes are described below and are shown in Figure 9.

1. Bricks consists of a set of $n+1$ parallelepipeds, $\frac{n}{2}$ of which are staggered with the other $\frac{n}{2}$. The observer is positioned such that the output size is $\Theta\left(n^{2}\right)$. The objective from choosing this scene is to find whether more than quadratic time or space would be needed.

2. Hidden Bricks consists of the same scene as Bricks, but the parallelepipeds are hidden from the observer by one larger parallelepiped. We wish to determine whether the algorithm takes time and space close to linear in the number of edges in the input for this type of scene.

3. Platforms consists of a set of slabs that are increasingly larger as seen from the observer. This scene will highlight an inherent weakness in using binary space partitioning.

4. Random consists of a set of cubes with random orientations. In this scene of linear-size output, we wish to determine the effect such random orientations would have on the time and space used.

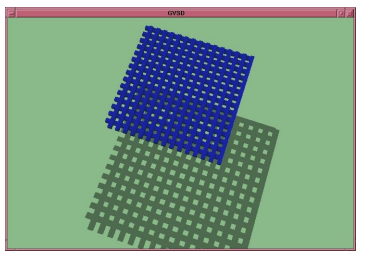

Bricks

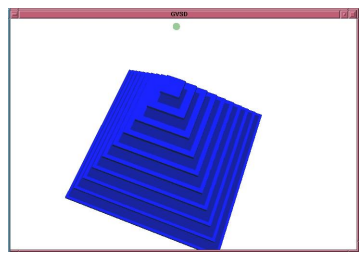

Platforms

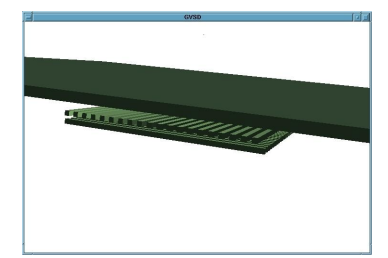

Hidden Bricks

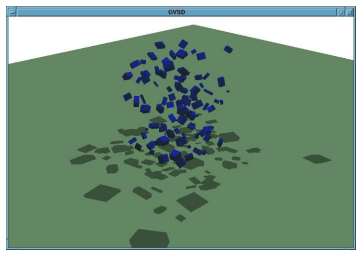

Random

Figure 9: The scenes used for the time and space experiments.

The time and space diagrams for each class of input are shown in Figures 10 and 11, respectively. 
Bricks

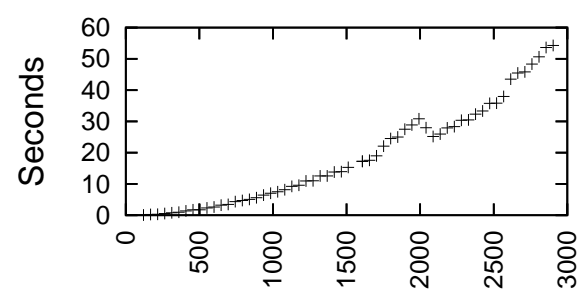

\#Edges

Platforms

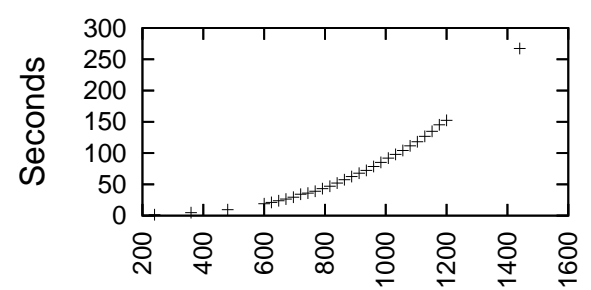

\#Edges
Hidden Bricks

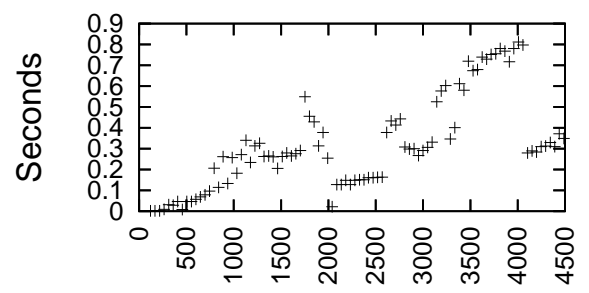

\#Edges

Random

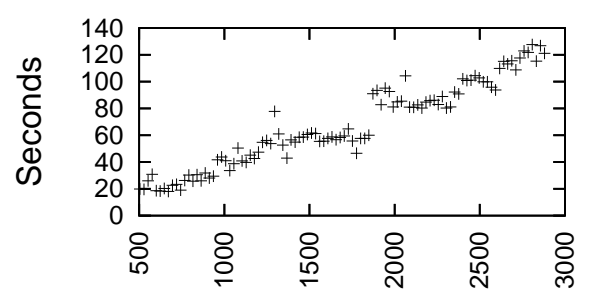

\#Edges

Figure 10: The time needed is shown as a function of the number of edges in the input.
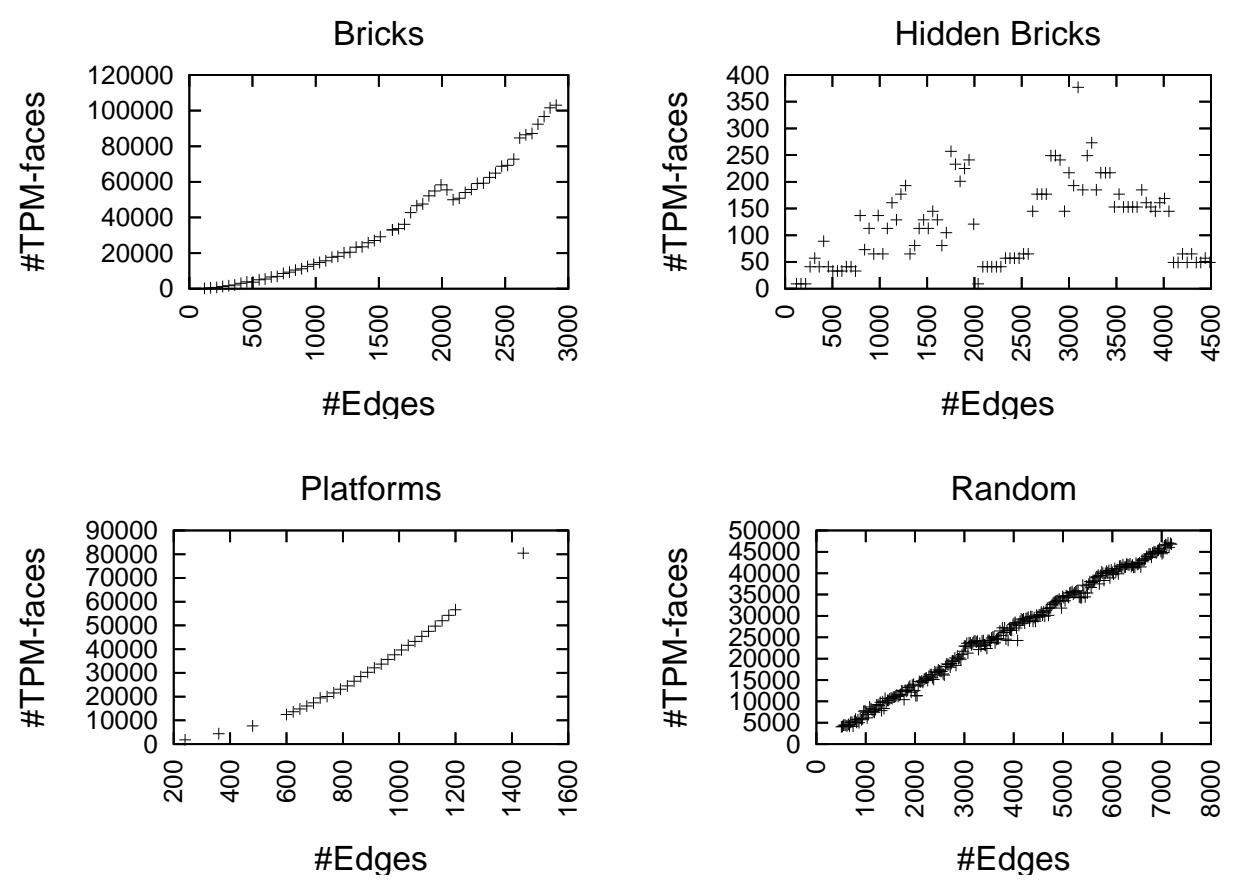

Figure 11: The space needed is shown as a function of the number of edges in the input.

The diagrams for the Hidden Bricks scene confirm that hidden intersections are never computed. In 
the Platforms scene, each parallelepiped is divided by partitioning planes formed by all edges nearer to the observer. Thus even though the output spherical map could be described using linear storage, the use of BSP trees results in an output of quadratic size.

Our implementation is available. All classes are parameterized and rely on the template mechanism of $\mathrm{C}++$ to delay the choice of the number type used for coordinates. An implementation that is both datageneric as well as algorithm-generic [2] is underway.

\section{Conclusion and Future Work}

The spherical visibility map is not an end in itself, but is only the main ingredient for constructing a multitude of systems. Our immediate objective is to determine whether the recently introduced canvas class in the firefox browser makes it possible to implement a javascript-based system that visualizes the spherical visibility map (looking from, not at, the observer). If implemented with a suitable user interface, such a system would rival Quicktime VR in its ability; it would effectively duplicate the latter in the vector domain. Scalable Vector Graphics (SVG) technologies are gradually gaining momentum and it is also interesting to investigate whether the small size of vector images would be appealing in combination with SVG since the bandwidth needed to relay vector images to, say, portable devices could be significantly smaller than the equivalent raster data.

The potential for user interface experiments for a reprojection using the SVM is wide open. The programmer or system designer may of course choose one type of projection [9] and seal the system, but it is more interesting to investigate whether a user interface can be developed that can capture more than one type of projection, seamlessly moving from oblique to fish-eye for example, to render a vector image.

The spherical visibility map may also potentially make it possible to tackle a large set of yet unstudied problems. The SVM can be projected on an arbitrary plane to produce a conventional vector image on a planar surface, but the color used to draw each polygon must be carefully computed. Images that use a single given color (a grayscale or an RGB triple) for each polygon are hard to understand since adjacent polygons of a cube, say, would not clearly delineate the difference in lighting. Also, determining a unique color for each polygon is not sufficient since the shading varies for points on the polygon. Raster algorithms avert this issue by computing shading on a per-pixel basis. A desirable shaded vector image would consist of a triangulation of the sphere into regions with an upper bound on the difference between the shading color used for any two adjacent triangles.

The term BSP tree is commonly used to refer both to a tree that stores polygons in interior leaves, which is used for depth order computation, as well as to a tree that stores partitioning planes in interior nodes and Boolean flags in leaves, which is used to define regular point sets. But no implementation is currently known that captures both versions of the BSP tree simultaneously. A generic implementation that captures both types of trees as well as other potential uses of recursive space partitioning would be advantageous. Also, an implementation that captures trees both in $2 \mathrm{D}$ and in $3 \mathrm{D}$ is also desirable. Our current implementation uses distinct structures for computing depth orders in 3D and for representing point sets on a sphere.

BSP trees are one of only four methods currently known for representing point sets in computing. The others are boundary representations (b-reps), Constructive Solid Geometry (CSG), and Nef polyhedra. Yet BSP trees are superior for our application since b-reps make it difficult to compute Boolean operations and both CSG and Nef polyhedra mandate the computation of neighborhoods, which result in intricate algorithms and implementations [35, 17, 50]. Since Nef polyhedra are capable of capturing non-manifold topologies whereas BSP trees only capture regular sets, it is conceivable that an application that requires the representation of a polygon visible "head on" (the observer lies in its plane) would require the use of Nef 
polyhedra. It is more likely, however, that one would want to discard the view of a lamina if its plane passes by the observer.

A natural question to ask is whether it is possible to extend the point sets representable by BSP trees [42] (in the plane, on a sphere, or in space) to capture non-manifold topologies. Such an extension could store lower-dimensional BSP trees in interior nodes to classify points on a partitioning plane, thus making it possible to define arbitrary point sets. Already a step in this direction has been taken [5].

The SVM's adjacency information contains the subset of the visibility skeleton [19] involving a given vertex. It would also be possible to use the SVM to generate a catalogue of shadow edges [27] if the locations of its topological changes for a moving viewer can be identified efficiently.

\section{Acknowledgment}

This work was supported by the NSERC grant The Practice of Object Space Computer Graphics.

\section{References}

[1] A. Appel. The notion of quantitative invisibility and the machine rendering of solids. In Proc. ACM National Conf., pages 387-393, 1967. also published in Rosalee Wolfe, ed., Seminal Graphics-Pioneering Efforts that shaped the Field, ACM Press, 1998, pp. 19-26.

[2] M. H. Austern. Generic Programming and the STL: Using and Extending the C++ Standard Template Library. Addison-Wesley, 1998.

[3] Daniel Azuma. GLOW. http://glow.sourceforge.net/.

[4] C. Bajaj. The algebraic degree of geometric optimization problems. Discrete Comput. Geom., 3:177-191, 1988.

[5] C. Baldazzi and A. Paoluzzi. Dimension-independent BSP (1): Section and interior to boundary mapping. Technical Report RT-DIA-26-97, Roma Tre, July 1997.

[6] U. Bartuschka, K. Mehlhorn, and S. Näher. A robust and efficient implementation of a sweep line algorithm for the straight line segment intersection problem. In Proc. Workshop on Algorithm Engineering, pages 124-135, 1997.

[7] B. G. Baumgart. A polyhedron representation for computer vision. In Proc. AFIPS Natl. Comput. Conf., volume 44, pages 589-596, 1975.

[8] J. Blinn. Fractional invisibility. IEEE CG\&A, November 1988. also available in Jim Blinn's Corner, A Trip Down the Graphics Pipeline, Chap. 10, Morgan Kaufman, 1996.

[9] I. Carlbom and J. Paciorek. Geometric projection and viewing transformations. ACM Computing Surveys, 10:465-502, 1978.

[10] CGAL Reference Manual, CGAL 3.1 edition. http://www.cgal.org/.

[11] N. Chin and S. Feiner. Near real-time shadow generation using BSP trees. In SIGGRAPH '89, pages 99-106, August 1989.

[12] N. Dadoun, D. G. Kirkpatrick, and J. P. Walsh. The geometry of beam tracing. In Proc. of the Symp. on Computational Geometry, pages 55-61, June 1985. the use of BSP trees and hierarchical bounding volumes for fast beam intersection testing.

[13] M. de Berg. Efficient algorithms for ray shooting and hidden surface removal. Ph.D. dissertation, Dept. Comput. Sci., Utrecht Univ., Utrecht, Netherlands, 1992. 
[14] M. de Berg, D. Halperin, M. H. Overmars, J. Snoeyink, and M. J. van Kreveld. Efficient ray shooting and hidden surface removal. Algorithmica, 12(1):30-53, July 1994.

[15] F. Dévai. Quadratic bounds for hidden line elimination. In Proc. 2nd Annu. ACM Sympos. Comput. Geom., pages 269-275, 1986.

[16] F. Dévai. An intersection-sensitive hidden-surface algorithm. In G. Marechal, editor, Eurographics '87, pages 495-502. North-Holland, August 1987.

[17] K. Dobrindt, K. Mehlhorn, and M. Yvinec. A complete and efficient algorithm for the intersection of a general and a convex polyhedron. In Proc. 3rd Workshop Algorithms Data Struct., volume 709 of Lecture Notes Comput. Sci., pages 314-324, 1993.

[18] S. E. Dorward. A survey of object-space hidden surface removal. Internat. J. Comput. Geom. Appl., 4:325-362, 1994.

[19] F. Durand. 3D Visibility: Analytical Study and Applications. PhD thesis, Université Joseph Fourier, Grenoble, France, 1999.

[20] A. Fabri, G.-J. Giezeman, L. Kettner, S. Schirra, and S. Schönherr. On the design of CGAL: a computational geometry algorithms library. Software-Practice and Experience, 30(11):1167-1202, September 2000.

[21] E. Flato, D. Halperin, I. Hanniel, O. Nechushtan, and E. Ezra. The design and implementation of planar maps in CGAL. ACM Journal of Experimental Algorithms, 5:13, 2000.

[22] J. D. Foley, A. van Dam, S. K. Feiner, and J. F. Hughes. Computer Graphics: Principles and Practice in C. Addison-Wesley, 2nd edition, 1995.

[23] S. Fortune. Topological beam tracing. In Sympos. on Comput. Geom., pages 59-68, 1999.

[24] W. R. Franklin. A linear time exact hidden surface algorithm. SIGGRAPH '80, 14(3):117-123, July 1980.

[25] H. Fuchs, Z. M. Kedem, and B. Naylor. On visible surface generation by a priori tree structures. SIGGRAPH '80, 14(3):124-133, July 1980.

[26] R. Galimberti and U. Montanari. An algorithm for hidden-line elimination. Commun. of the ACM, 12(4):206211, April 1969.

[27] S. Ghali. A Geometric Framework for Computer Graphics Addressing Modeling, Visibility, and Shadows. PhD thesis, Department of Computer Science, University of Toronto, 1999.

[28] M. T. Goodrich. A polygonal approach to hidden-line and hidden-surface elimination. CVGIP: Graph. Models Image Process., 54(1):1-12, 1992.

[29] D. Gordon and S. Chen. Front-to-back display of BSP trees. IEEE Computer Graphics \& Appl., pages 79-85, Sep 1991.

[30] L. J. Guibas and J. Stolfi. Primitives for the manipulation of general subdivisions and the computation of Voronoi diagrams. ACM Trans. on Graphics, 4(2):74-123, April 1985.

[31] P. S. Heckbert and P. Hanrahan. Beam tracing polygonal objects. SIGGRAPH '84, 18-3:119-127, July 1984.

[32] M. J. Katz, M. H. Overmars, and M. Sharir. Efficient hidden surface removal for objects with small union size. Comput. Geom. Theory Appl., 2:223-234, 1992.

[33] N. Kitsios and A. Tsakalidis. Space reduction and an extension for a hidden line elimination algorithm. Comput. Geom. Theory and Appl., 6, 1996.

[34] P. P. Loutrel. A solution to the hidden-line problem for computer drawn polyhedra. IEEE Trans. Comput., C-19:205-213, 1970.

[35] M. Mäntylä. An Introduction to Solid Modeling. Computer Science Press, Rockville, MD, 1988. 
[36] M. J. Mäntylä and R. Sulonen. GWB: A solid modeler with Euler operators. IEEE Computer Graphics \& Appl., 2(5):17-31, 1982.

[37] M. McKenna. Worst-case optimal hidden-surface removal. ACM Trans. on Graphics, 6:19-28, 1987.

[38] D. E. Müller and F. P. Preparata. Finding the intersection of two convex polyhedra. Theoret. Comput. Sci., 7:217-236, 1978.

[39] K. Mulmuley. An efficient algorithm for hidden surface removal. SIGGRAPH '89, 23(3):379-388, 1989.

[40] B. Naylor. Binary space partitioning trees as an alternative representation of polytopes. Computer-Aided Design, 22(4):250-252, 1990.

[41] B. Naylor. Partitioning tree image representation and generation from $3 \mathrm{~d}$ geometric models. In Proceedings of Graphics Interface '92, pages 201-212, May 1992.

[42] B. Naylor, J. A. Amanatides, and W. Thibault. Merging BSP trees yields polyhedral set operations. SIGGRAPH '90, 24(4):115-124, August 1990.

[43] M. Newell, R. Newell, and T. Sancha. A new solution to the hidden surface problem. In Proc. ACM Annual Conf., pages 443-448, 1972.

[44] A. Ricci. An algorithm for the removal of hidden lines in 3d scenes. Comput. J., 14(4):375-377, 1971.

[45] L. Roberts. Machine perception of 3-D solids. Technical report, MIT, Lincoln Lab., Cambridge, 1963.

[46] A. Schmitt. On the time and space complexity of certain exact hidden line algorithms. Report 24/81, Fakultät Inform., Univ. Karlsruhe, Karlsruhe, West Germany, 1981.

[47] A. Schmitt. Time and space bounds for hidden line and hidden surface algorithms. In Proc. Eurographics 81, pages 43-56, Amsterdam, Netherlands, 1981. North-Holland.

[48] R. Schumacher, R. Brand, M. Gilliland, and W. Sharp. Study for applying computer-generated images to visual simulation. Technical report, Air Force Human Resources Laboratory. Training Research Division., 1969.

[49] S. Sechrest and D. P. Greenberg. A visible polygon reconstruction algorithm. ACM Trans. on Graphics, 1(1):2542, January 1982.

[50] M. Seel. Planar Nef Polyhedra and Generic Higher-Dimensional Geometry. PhD thesis, Universität des Saarlandes, August 2001.

[51] M. Sharir and M. Overmars. A simple output-sensitive algorithm for hidden-surface removal. ACM Trans. on Graphics, 11:1-11, 1992.

[52] I. E. Sutherland and G. W. Hodgman. Reentrant polygon clipping. Commun. of the ACM, 17:32-42, 1974.

[53] I. E. Sutherland, R. F. Sproull, and R. A. Schumacker. A characterization of ten hidden-surface algorithms. ACM Computing Surveys, 6(1):1-55, March 1974.

[54] S. J. Teller and C. H. Séquin. Visibility preprocessing for interactive walktroughs. SIGGRAPH '91, 25(4):61-69, 1991.

[55] W. C. Thibault. Application of Binary Space Partitioning Trees to Geometric Modeling and Ray-Tracing. PhD thesis, Georgia Institute of Technology, 1987.

[56] W. C. Thibault and B. F. Naylor. Set operations on polyhedra using binary space partitioning trees. SIGGRAPH '87, 21(4):153-162, 1987.

[57] N. Tsingos, T. Funkhouser, A. Ngan, and I. Carlbom. Modeling acoustics in virtual environments using the uniform theory of diffraction. SIGGRAPH '01, pages 545-552, 2001.

[58] J. Warnock. A hidden-surface algorithm for computer generated half-tone pictures. Technical Report TR 4-15, NTIS AD-733 671, University of Utah, Computer Science Department, 1969.

[59] K. Weiler and P. Atherton. Hidden surface removal using polygon area sorting. SIGGRAPH '77, 11(2):214-222, 1977. 